\title{
Generalised osteitis fibrosa cystica due to secondary hyperparathyroidism in chronic kidney disease
}

\author{
S K Shrestha, ${ }^{1}$ A Tayal ${ }^{2}$ \\ ${ }^{1}$ Lecturer,Department of Orthopaedics, ${ }^{2}$ Associate.Professor, Departrment of Radiodiagnosis, College of \\ Medical Sciences, Teaching Hospital, Bharatpur, Nepal
}

\begin{abstract}
Secondary hyperparathyroidism is a frequent complication of patients with chronic kidney disease and is characterized by excessive serum parathyroid hormone levels and an imbalance in calcium and phosphorus metabolism.
\end{abstract}

Secondary hyperparathyroidism is the leading cause of renal osteodystrophy and bone disease. Osteitis fibrosa cystica, the classic and former most common osteodystrophy, is mainly caused by high bone turnover secondary to high levels of circulating PTH. Its pathophysiology is mainly due to hyperphosphatemia and vitamin D deficiency and resistance.

This condition has a high impact on the mortality and morbidity of dialysis patients Hyperparathyroidism develops early in the course of CKD and becomes more prominent as kidney function declines. However recently, with the technical development of imaging and laboratory screening methods, hypercalcemia due to primary or secondary hyperparathyroidism can often be detected early; as a result the frequency of osteitis fibrosa cystica has declined.

Key words: Chronic kidney disease, secondary hyperparathyroidism, osteitis fibrosa cystica.

\section{Introduction}

Secondary hyperparathyroidism (SHPT) is a common complication of chronic kidney disease (CKD), defined by elevated levels of PTH, and is associated with abnormalities in calcium, phosphorus and vitamin D metabolism. ${ }^{1}$ Secondary hyperparathyroidism (SHPT) develops early in chronic kidney disease (CKD), before dialysis is required. Consequently, most patients with chronic kidney disease stage 5 or end-stage renal disease (ESRD) have elevated parathyroid hormone

Dr. Sundar K. Shrestha

Email: sundarpuspa@gmail.com and secondary hyperparathyroidism of variable severity. Vitamin D deficiency is common and is underdiagnosed. Renal osteodystrophy and progressive vascular calcifications are the 2 major outcomes of SHPT. ${ }^{2,3}$

The symptoms of the disease are the consequences of both the general softening of the bones and the excess calcium in the blood and include bone fractures, kidney stones, nausea, peptic ulcers, appetite loss, and weight loss_-'bones, stones, abdominal groans and psychic overtones". 4 
Research has shown that CKD patients who are classified as Stage 3, Stage 4, or Stage 5 are at risk for, or may already have developed, SHPT. The early identification and treatment of SHPT is crucial to preventing or controlling the consequences of this complication.

The term renal osteodystrophy represents a variety of bone disorders caused by chronic renal failure (CRF). Renal osteodystrophy is sometimes called "the silent crippler"; affected patients may be completely asymptomatic. Symptoms, including bone and joint pain and bone deformation and fractures, are more frequent during the late stages of the disease. The most common abnormality is the Osteitis Fibrosa Cystica, with extensive bone marrow fibrosis and increased osteoclastic bone resorption. ${ }^{5}$ It is a reparative cellular process, rather than a neoplastic process. These lesions are termed "Brown Tumour" due to the presence of old hemorrhage in the lesion and its reddish color.

Bone resorption occurs because of increased osteoclastic activity and affects all bone surfaces at different skeletal sites. It may be subperiosteal, intracortical, endosteal, trabecular, subchondral, subligamentous or subtendinous. ${ }^{6}$ Fragility fractures sometimes occur at the sites of brown tumors. These lesions are caused by rapid osteoclastic activity and peritrabecular fibrosis, and are usually well-defined purely lytic lesions with the cortex thinned and expanded but not penetrated. Since they are usually painless, the clinical diagnosis is commonly made when the patient presents a fracture. Brown tumors appear mostly at the pelvis, ribs, clavicles, mandible and extremities. $^{7}$

\section{Pathophysiology of Osteitis Fibrosa Cystica}

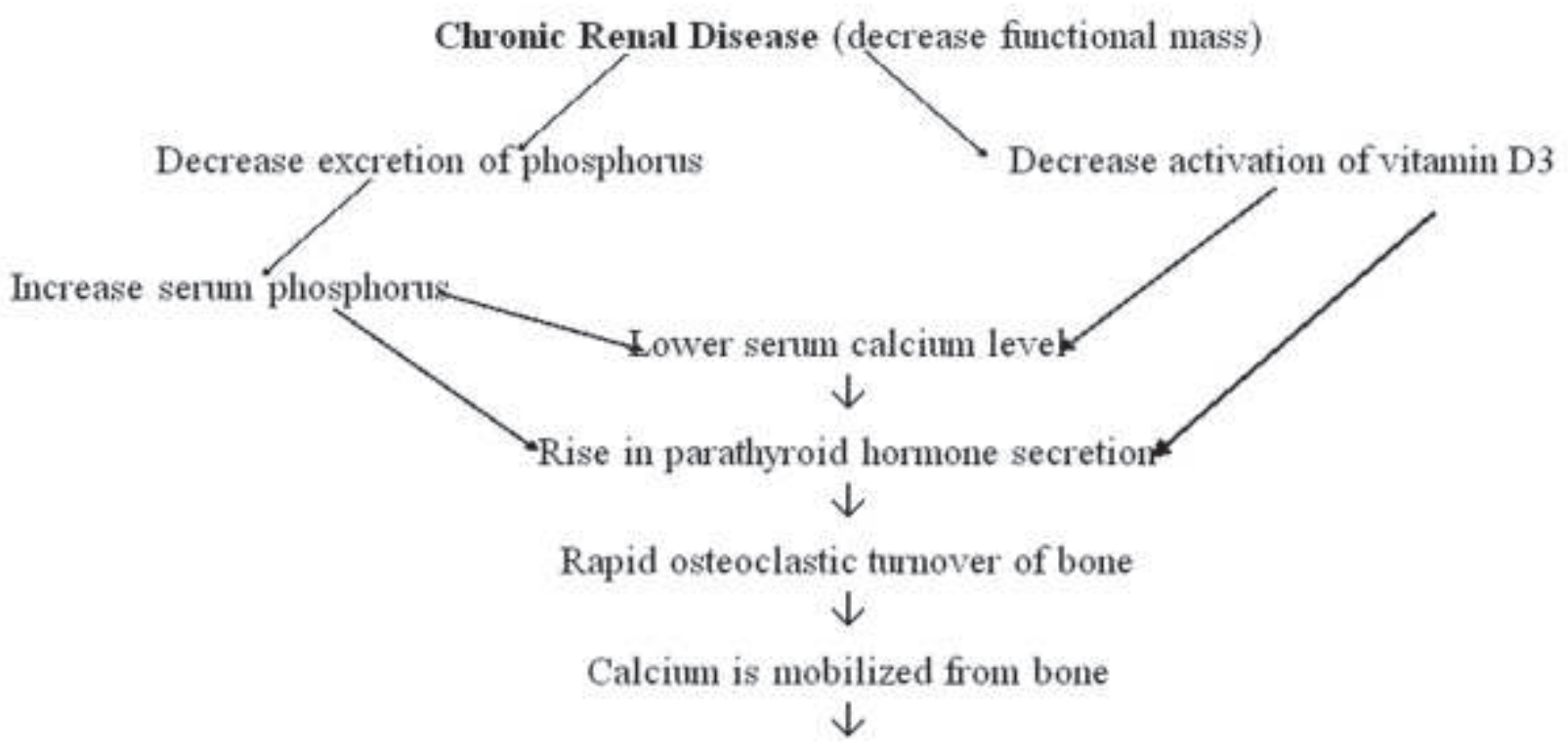

Normal or Increase serum calcium level

$\downarrow$

In regions with rapid bone loss; haemorrhage, reparative granulation tissue and vascular; proliferating fibrous tissue replace the normal marrow content

\section{Osteitis Fibrosa Cystica}


SHPT secondary to CKD is an overproduction of PTH caused by several changes that occur in bone and mineral metabolism as a result of decreased kidney function. The first changes that usually occur with declining kidney function involve the deficiency of activated vitamin $\mathrm{D}$ and an increase in phosphorus excretion by the remaining functional nephrons. Both of these changes stimulate an increase in PTH synthesis and secretion.

\section{Case report}

45-year-old female presented with mutiple bony prominences over shins of both legs and left clavicle for 5-6months with generalized body pain. Also had a shortness of breath and easy fatiguability for one week. There was a history of weight loss and loss of appetite. Examination revealed multiple bony swelling over both shin of tibia, single bony swelling over medial third of left clavicle. It was hard, non tender and fixed swelling. Patient also had pallor, edema, palpable right supraclavicular lymph node.

Radiographs revealed generalized thinning of cortices and multiple cystic lesions of the both tiba (Fig. A\&B), subperiosteal erosion of both femur diaphysis (Fig. C) and osteolytic lesions of left ulna, left clavicle and left third metacarpal bone (Fig. D, E and F).

Computed Tomography of chest show lytic lesions of multiple ribs (Fig. $\mathrm{G}$ and $\mathrm{H}$ ).

Blood parameters showed; calcium: $7.4 \mathrm{mg} / \mathrm{dl}$, PTH: 1603 pg/mL, Phosphorus: 6.5 mg/dl, Hb\%: 6.8, RBC: 1.51, WBC: 11.01, Platelet: 1.87, ESR: 50, Na: 148 meq/l, K: 4.6 meq/dl, Urea: 220 mg/dl, Creatinine: 6 mg/dl. SGOT: 20.4, SGPT: 26.4, Alkaline

phosphatase: 313.1, Urinalysis; $\mathrm{PH}$ : acidic, Albumin: +++, Sugar: + WBC: 1-2, RBC: Plenty

Incision and curettage of the right tibia lesion was done, and histopathology reported it as brown tumor of hyperparathyroidism (Fig. I).

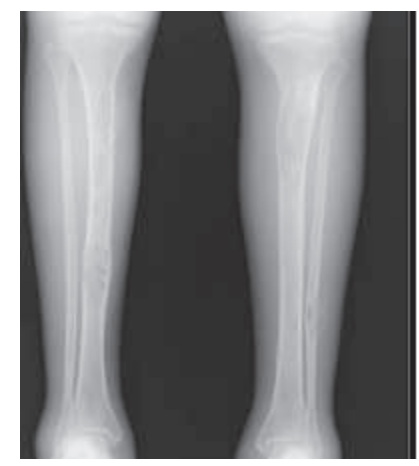

A

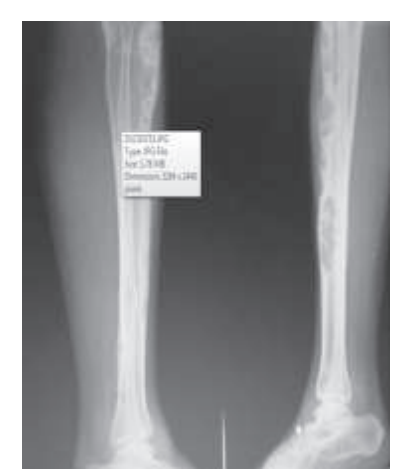

B

Fig: A \& B: AP and Lateral Radiograph of both tibia and fibula showing multiple OFC lesions.

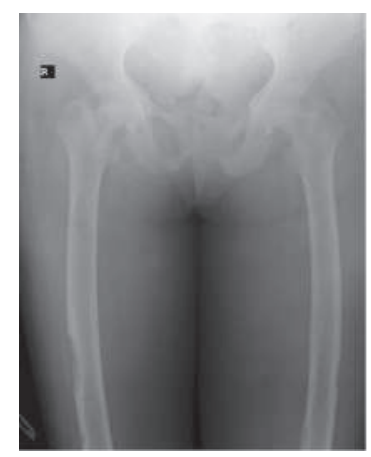

C

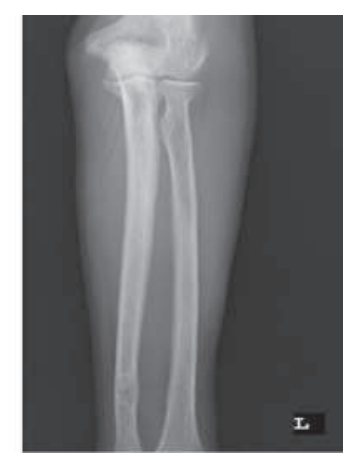

$\mathrm{D}$

Figure C: AP Radiograph showing subperiosteal Erosions (OFC) of both Femur Diaphysis.

Figure D: AP Radiograph showing OFC lesion of left Ulna Diaphysis (Distal Third)

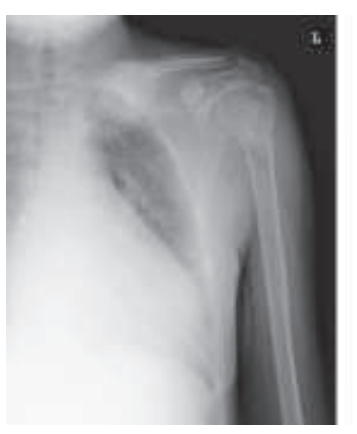

$\mathrm{E}$

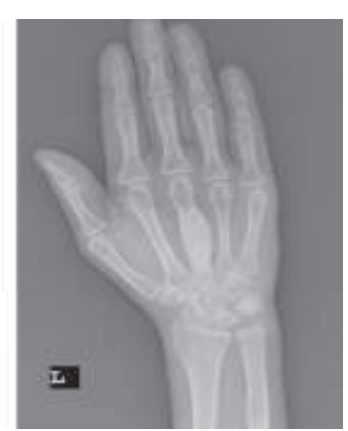

F 
Figure E \& F: AP Radiograph showing OFC Lesion of a Left Clavicle and Third Metacarpal of Left Hand

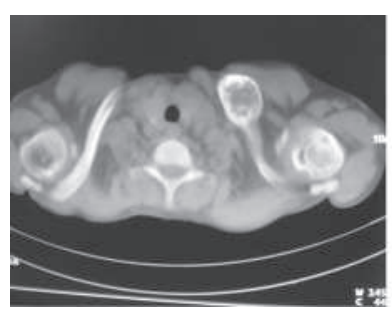

$\mathrm{G}$

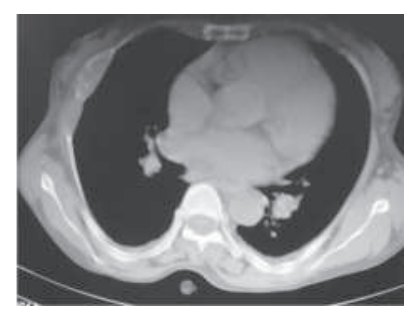

$\mathrm{H}$
Figure G \& H: Chest Computed Tomography, Axial Scan showing OFC lesion of Ribs.

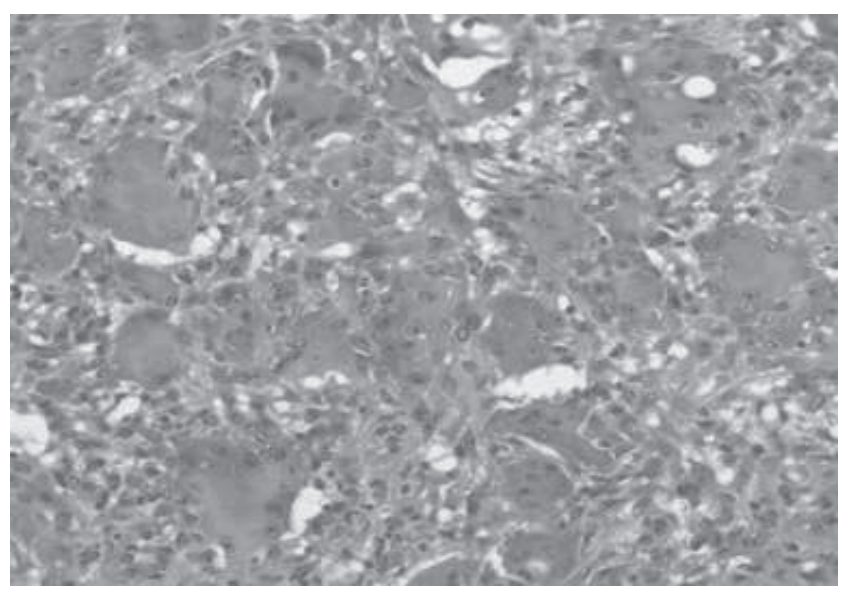

Fig. I. This section shows diffuse proliferation of osteoclast-type giant cells intermixed osteoblastic proliferation, and neovascularizaion, suggestive of osteitis fibrosa cystica $(\mathrm{H} \& \mathrm{E}, \times 200)$.

\section{Discussion}

Evidence suggests that the alterations in serum PTH and mineral metabolism in SHPT have important consequences for haemodialysis patients. Increased calcium and phosphorus concentrations are key contributors to SHPT-associated all-cause and cardiovascular (CV) mortality and bone disease. ${ }^{\mathbf{8 , 9}}$

Osteitis fibrosa cystica was first described in the nineteenth century by von Recklinghausen; it presents as the end stage findings of hyperparathyroidism. However recently, with the technical development of imaging and laboratory screening methods, hypercalcemia due to primary or secondary hyperparathyroidism can often be detected early; as a result the frequency of osteitis fibrosa cystica has declined. ${ }^{10,11}$

However, it is not a true neoplasm but rather a reactive osteolytic lesion of bone and may mimic other diseases such as giant cell tumors, multiple bone metastasis or multiple myeloma. ${ }^{12,13}$

Osteitis fibrosa cystica appears as a well-defined expansile osteolytic lesion with osteopenia on plain radiographs, and as a well enhanced mass on $\mathrm{CT}$ and MRI T2-weighted imaging, and is isodense on T1weighted images. ${ }^{14}$

The histopathology shows diffuse proliferation of osteoclastic multinucleated giant cells intermixed with fibrocellular proliferation and hemorrhagic foci, changes that may be related to a microfracture undergoing organization with the release of hemosiderin and appears as a reddish brown mass. However, the histopathological feature alone cannot confirm the diagnosis because other giant cell lesions such as giant cell granuloma and aneurismal bone cysts have similar histological features. ${ }^{5}$

Recent studies suggest that optimal management of mineral homeostasis (calcium, phosphorus, and calcium-phosphorus product) may reduce morbidity and mortality in patients with SHPT.

Prevention and treatment of SHPT requires control of serum phosphate and restoration of $1,25(\mathrm{OH}) 2 \mathrm{D} 3$ levels. The overall goal is to restore the PTH-calcium- 
vitamin D endocrine feedback loop and re-establish mineral homeostasis. ${ }^{15}$ Thus, early diagnosis of SPHT and timely intervention is the key to restoring feedback mechanism and prevention of complications.

Management of hyperphosphatemia among CKD patients remains a major challenge for nephrologists. Serum phosphorus plays a significant role in PTH regulation, and animal studies have shown that increased dietary phosphorus can increase PTH mRNA expression. ${ }^{16}$

For patients with chronic kidney disease, the National Kidney Foundation (NKF) published clinical practice guidelines as part of its Kidney Disease Outcomes Quality Initiative (KDOQI). In general, it has been recommended to reduce parathyroid hormone levels to within a range that supports normal bone turnover and minimizes ectopic calcification.

The following is a list of current nonsurgical treatment options for management of secondary hyperparathyroidism in chronic kidney disease: ${ }^{17}$

- Dietary phosphate restriction can be initiated if parathyroid hormone is elevated despite sufficient 25-hydroxyvitamin $\mathrm{D}(>30 \mathrm{ng} / \mathrm{mL})$.

- Phosphate binders can be used if hyperphosphatemia persists despite dietary phosphate restriction. These include calciumbased phosphate binders such as calcium carbonate or calcium acetate and non-calciumbased phosphate binders such as sevelamer hydrochloride or lanthanum carbonate.

- Calcium supplementation should be limited to less than $2 \mathrm{~g} / \mathrm{d}$.
- Vitamin D and its analogs may be used, including calcitriol and analogs of calcitriol, including paricalcitol, doxercalciferol, maxacalcitol, and falecalcitriol.

Indications for surgery include bone pain or fracture, pruritus, calciphylaxis (see Related Disorders), and extraskeletal nonvascular calcifications with elevated parathyroid hormone levels despite appropriate medical therapy. Parathyroidectomy can be considered in patients with severe hyperparathyroidism (persistent serum levels of intact parathyroid hormone greater than $800 \mathrm{pg} / \mathrm{mL}$ [88.0pmol/L]), associated with hypercalcemia and/or hyperphosphatemia that is refractory to medical therapy. ${ }^{18}$

\section{Conclusion}

CKD is associated with multiple complications including SHPT, the resulting condition is associated with cardiovascular and bone diseases resulting in high morbidity and mortality.

Although bone histology remains the best method for differentiating between the forms of renal osteodystrophy, serum levels of parathyroid hormone (PTH) are commonly used for diagnosing secondary hyperparathyroidism and OFC. In patients with end stage renal disease (ESRD) and chronic hemodialysis, PTH serum levels are considered adequate between 150 and $300 \mathrm{pg} / \mathrm{ml}$. Four to eightfold elevations of PTH are predictive for high-turnover bone disease and levels above this are strongly correlated with OFC..$^{\mathbf{1 9}, 20}$

Timely supplementation with vitamin D and vitamin D analogs may prevent development of SHPT and its complications. Early diagnosis and appropriate 
S K Shrestha et al, Generalised osteitis fibrosa cystica

treatment paradigms are cornerstones for any effective strategy to maximize the care available to patients with CKD.

Medical treatment of secondary hyperparathyroidism is successful in most patients. Patients who require parathyroidectomy have approximately a $10 \%$ risk of recurrent or persistent disease.

\section{References}

1. Slatopolsky E, Delmez JA. Pathogenesis of secondary hyperparathyroidism. Am J Kidney Dis 1994;23 (2).

2. Fukagawa M, Kazama JJ, Kurokawa K. Renal osteodystrophy and secondary hyperparathyroidism. Nephrol Dial Transplant 2002; 17:10.

3. Moe SM. Current issues in the management of secondary hyperparathyroidism and bone disease. Perit Dial Int 2001;21: 3.

4. J. F. Murray, Ed., Primer on the Metabolic Bone Diseases and Disorders of Mineral Metabolism, Raven Press, New York, NY, USA, 2nd edition, 1993.

5. Kemp AM, Bukvic M, Sturgis CD. Fine needle aspiration diagnosis of osteitis fibrosa cystica (brown tumor of bone): a case report. Acta Cytol. 2008;52(4):471-4

6. Jevtic V. Imaging of renal osteodystrophy. Eur J Radiol 2003;46: 85-95.

7. Fineman I, Johnson JP, Di-Patre PL et al. Chronic renal failure causing brown tumors and myelopathy. Case report and review of pathophysiology and treatment. J Neurosurg 1999; 90: 242-6.
8. Block GA, Klassen PS, Lazarus JM et al. Mineral metabolism, mortality, and morbidity in maintenance hemodialysis. J Am Soc Nephrol 2004; 15: 2208-18.

9. Elder G. Pathophysiology and recent advances in the management of renal osteodystrophy. $J$ Bone Miner Res 2002; 17: 2094-2105.

10. Hsieh MC, Ko JY, Eng HL. Pathologic fracture of the distal femur in osteitis fibrosa cystica simulating metastatic disease. Arch Orthop Trauma Surg. 2004;124(7):498-501.

11. Guney E, Yigitbasi OG, Bayram F et al. Brown tumor of the maxilla associated with primary hyperparathyroidism. Auris Nasus Larynx. 2001;28(4):369-72.

12. Hsieh MC, Ko JY, Eng HL. Pathologic fracture of the distal femur in osteitis fibrosa cystica simulating metastatic disease. Arch Orthop Trauma Surg. 2004;124(7):498-501.

13. Atabek ME, Pirgon O, Sert A et al. Extensive brown tumors caused by parathyroid adenoma in an adolescent patient. Eur J Pediatr. 2008;167(1):117-9.

14. Takeshita T, Tanaka H, Harasawa A, Kaminaga $\mathrm{T}$, Imamura $\mathrm{T}$, Furui S. Brown tumor of the sphenoid sinus in a patient with secondary hyperparathyroidism: CT and MR imaging findings. Radiat Med. 2004 Jul-Aug;22(4):265-8.

15. Locatelli F, Cannata-Andia JB, Drueke TB, et al. Management of disturbances of calcium and phosphate metabolism in chronic renal insufficiency, with emphasis on the control of hyperphosphataemia. Nephrol Dial Transplant 2002;17 (5).

16. Naveh-Many T, Rahamimov R, Livni N, et al. Parathyroid cell proliferation in normal and 
chronic renal failure rats. The effects of calcium, phosphate, and vitamin D. J Clin Invest 1995;96 (4).

17. Andress DL, Norris KC, Coburn JW, et al. Intravenous calcitriol in the treatment of refractory osteitis fibrosa of chronic renal failure. N Engl J Med 1989;321 (5).

18. Rothmund M, Wagner PK, Schark C. Subtotal parathyroidectomy versus total parathyroidectomy and autotransplantation in secondary hyperparathyroidism: a randomized trial. World J Surg. Nov-Dec 1991;15(6):745-50.

19. Schwarz C, Sulzbacher I, Oberbauer R. Diagnosis of renal osteodystrophy. Eur J Clin Invest 2006; 36: 13-22.

20. Torres A, Lorenzo V, Hernández D, et al. Bone disease in predialysis, hemodialysis, and CAPD patients: evidence of a better bone response to PTH. Kidney Int 1995; 47: 1434-1442. 